Известия НАН Армении, Физика, т.57, №1, с.19-29 (2022)

УДК 621.384

DOI:10.54503/0002-3035-2022-57.1-19

\title{
ТЕСТОВЫЕ ПУЧКИ ЭЛЕКТРОНОВ НА БАЗЕ ЛИНЕЙНОГО УСКОРИТЕЛЬНОГО КОМПЛЕКСА ЛУЭ-75 НАЦИОНАЛЬНОЙ НАУЧНОЙ ЛАБОРАТОРИИ ИМ. А.И. АЛИХАНЯНА
}

\author{
А.С. АКОПЯН ${ }^{1 *}$, Г.О. МАРУКЯН ${ }^{1}$, Г.Г. АКОПЯН ${ }^{1}$, А.З. БАБАЯН ${ }^{1}$, \\ Л.Р. ВАГРАДЯН ${ }^{1}$, В. БАРАНОВ ${ }^{2}$, Ю.И. ДАВЫДОВ ${ }^{2}$, А.В. КРАСНОПЕРОВ ${ }^{2}$, \\ А. СИМОНЕНКО ${ }^{2}$, В. ТЕРЕЩЕНКО ${ }^{2}$, Г.Т. ТОРОСЯН ${ }^{1,2}$, Г.Г. ЗОГРАБЯН ${ }^{1}$, \\ Г.М. АЙВАЗЯН ${ }^{1}$, Г.С. ВАРДАНЯН ${ }^{1}$, А.К. ПАПЯН ${ }^{1}$
}

\footnotetext{
${ }^{1}$ Национальная научная лаборатория им. А.И. Алиханяна, Ереван, Армения

${ }^{2}$ Объединенный институт ядерных исследований, Дубна, Россия

*e-mail: ashothako@yerphi.am

(Поступила в редакцию 21 июля 2021 г.)
}

\begin{abstract}
На базе линейного ускорителя электронов ЛУЭ-75 Национальной научной лаборатории им. А.И. Алиханяна разработана и применена методика получения управляемых первичных электронных пучков с интенсивностью (1020) электронов в секунду в диапазоне энергий (15-75) МэВ для калибровки детекторов элементарных частиц. Совместные работы с Лабораторией ядерных проблем им. В.П. Джелепова ОИЯИ (Дубна, РФ) показали работоспособность методики.
\end{abstract}

\section{1. Введение}

Линейный ускорительный комплекс ЛУЭ-75 Национальной научной лаборатории им. А.И. Алиханяна (ННЛА, Ереванский физический Институт ЕрФИ) до 2008 года служил в качестве инжектора ереванского электронно-кольцевого ускорителя АРУС (Армянский Ускоритель), в свое время крупнейшего в Советском Союзе электронного синхротрона с энергией до 6.1 ГэВ. ЛУЭ-75 в настоящее время продолжает успешно работать в автономном режиме. Это четырехсекционный резонансный ускоритель S-диапазона на бегущей волне. Он обеспечивает ускорение электронов в диапазоне энергий (10-75) МэВ при токах в импульсе до (150-200) мА, что соответствует среднему току до 10 мкА (без коллимации) в зависимости от длительности макроимпульса и энергии; на номинальных энергиях ширина энергетического спектра (FWHM) порядка 2\%. Подробно параметры линейного ускорительного комплекса ЛУЭ-75, включающего собственно линейный ускоритель и тракт транспортировки с параллельным переносом (рис.1), расположенный в кольцевом зале синхротрона, представлены в статье [1]. ЛУЭ-75 является единственным действующим ускорителем этого 


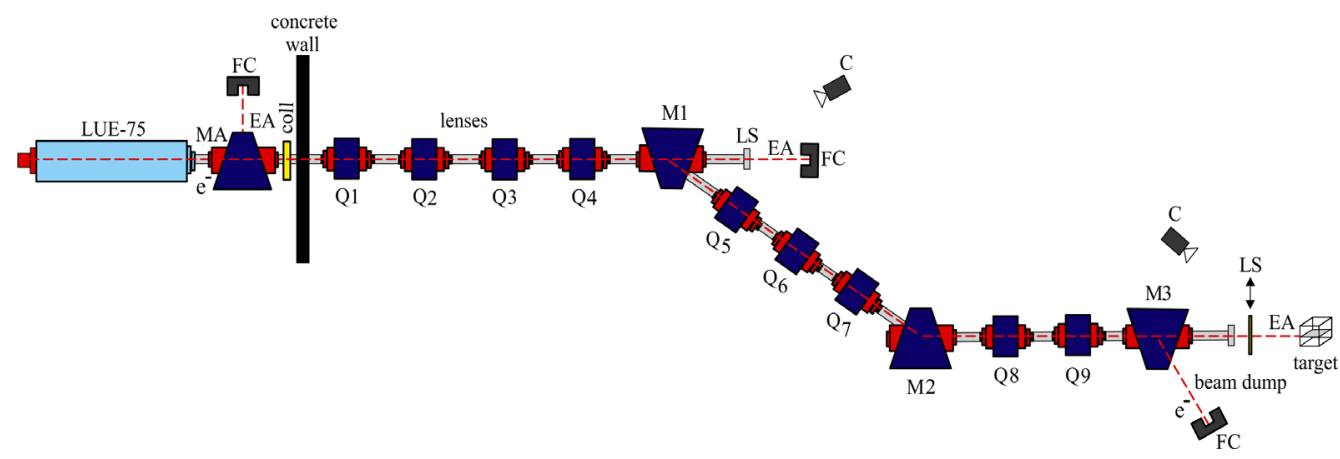

Рис.1. Упрощенная схема тракта транспортировки пучка с параллельным переносом: FC - цилиндр Фарадея; MA - анализирующий магнит; coll - коллиматор на выходе ускорителя; М1, М2 - магниты параллельного переноса; M3 - магнит-beam dump; Q1...Q9 - квадрупольные линзы; ЕА - экспериментальная зона; LS - люминофорный экран; C - камера.

класса в регионе. На нем проводят эксперименты исследовательские группы Армении, а также осуществляются совместные с научными группами из других стран проекты по решению фундаментальных вопросов ядерной физики низких энергий и прикладных научно-методических задач [2].

В последние годы были выполнены реновационно-восстановительные работы, целью которых было расширение возможностей ускорителя. В частности, в 2014-2015 гг. была разработана и в дальнейшем усовершенствована методика получения уникальных электронных пучков экстремально низкой интенсивности

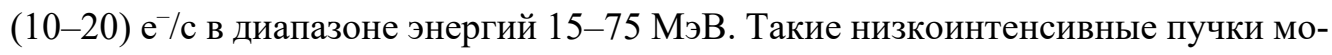
гут быть использованы для изучения отдельных событий, дифракционных задач, исследования излучения электронов в монокристаллах, изучения биологических эффектов ионизирующей радиации в малых дозах, в нанотехнологиях и др. В 2015-2019 гг. на ЛУЭ-75 такие пучки успешно применялись в качестве тестовых с числом одноэлектронных событий в импульсе более 70\% для калибровки кристаллических сцинтилляционных детекторов элементарных частиц в рамках совместных работ ННЛА (Ереван) - ОИЯИ (Дубна). Тестирование прототипа электромагнитного калориметра в виде матрицы из девяти нелегированных кристаллов CsI для эксперимента Mu2e [3], готовящегося в Фермилаб (США), показало эффективность методики. Сотрудничество по этим работам послужило мотивацией для постановки задачи, решение которой описывается ниже.

\section{2. Тестовые пучки предельно низкой интенсивности}

Высокочувствительные детекторы не могут быть исследованы или калиброваны пучками большой интенсивности во избежание радиационного разрушения, увеличения времени послесвечения, а также явления фосфоресценции [4]. Малоинтенсивные же излучения от источников естественного происхождения от радиоактивных изотопов и космические лучи - используются чаще в 
качестве индикаторных для проверки работоспособности как сцинтилляционных датчиков, так и регистрирующей аппаратуры на фиксированной энергии частиц. Космические мюоны используются не только для этих целей, но и для оценки временного и пространственного разрешения сцинтилляционных счетчиков, как это описано в работе [5].

В последние годы на ЛУЭ-75 совместно с исследовательской группой из ОИЯИ (Дубна, РФ) проводились методические работы по энергетической калибровке матрицы кристаллов CSI в диапазоне энергий 15-75 МэВ пучками интенсивностью (10-20) е $\mathrm{e}^{-} / \mathrm{c}$.

Управляемые электронные пучки экстремально низкой интенсивности получают в нескольких научных центрах [6,7]. Востребованный диапазон энергий электронов от 10-15 до 75 МэВ, который был необходим для калибровки кристаллов, ко времени проведения описываемых работ был предоставлен линейным ускорителем ЛУЭ-75.

Отметим, что впервые чрезвычайно слабый поток одиночных поочередно летящих электронов интенсивностью $4.2 \cdot 10^{3} \mathrm{e}^{-} / \mathrm{c}$, ускоренных в магнитном электронном микроскопе просвечивающего типа до энергии 72 кэВ, был получен в опытах по дифракции, описанных в известной работе [7].

В зависимости от типа, конструкции и возможностей ускорителя, решения такой задачи могут оказаться различными. Существует два способа получения таких электронных пучков: конвертерный метод и непосредственное получение первичного пучка сверхнизкой интенсивности $[5,6]$.

В 2014 г. была поставлена задача разработать методику получения тестовых пучков экстремально низкой интенсивности на основе ускорительного комплекса ЛУЭ-75 с использованием имеющейся в наличии техникотехнологической базы ННЛА (ЕрФИ) без внесения существенных изменений в конструкцию ускорителя. Было решено получить первичные контролируемые пучки предельно низкой интенсивности, используя благоприятные для поставленной цели конструктивные возможности ускорительного комплекса - наличие тракта транспортировки с параллельным переносом пучка, расположенного в синхротронном зале вдали от помещения ЛУЭ-75 за его радиационно-защитной стеной (рис.1). Проведенное исследование в зоне измерений показало практически отсутствие влияния электромагнитных помех и наводок от электрорадиотехнических устройств линейного ускорителя на измерительную аппаратуру, что важно для проведения прецизионных экспериментов.

Получение таких слабых интенсивностей усложняется также темновыми токами ускорителя, создающими проблемы для экспериментаторов: увеличение радиационного фона, повышение тепловыделения на стенках ускоряющих волноводов и пучкопровода, потеря части полезного пучка из-за наведенных паразитных полей и т.д. При получении пучков сверхмалых интенсивностей темновые токи ограничивают интенсивность полезного пучка снизу, поэтому выявление и устранение причин их возникновения является принципиальным. Они могут появиться по разным причинам: из-за СВЧ пробоев в ускоряющих секциях, ионизации остаточного в секциях газа при столкновении с электронами, 
автоэмиссии с поверхности металлической вакуумной камеры. Возникающие в этих процессах электроны могут попасть в благоприятную фазу ускоряющей волны и появиться на выходе ускорителя наряду с полезным пучком. В отличие от последнего, темновые токи носят случайный характер и неуправляемы: их энергия зависит от того, на каком участке ускоряющей структуры они возникли.

Измерения темновых токов с использованием электрометрической аппаратуры и магнитного анализатора с цилиндром Фарадея на выходе показали, что основным источником этих токов являлась начальная часть ускорителя: их интенсивность резко увеличивалась при повышении СВЧ-мощности в инжекторной секции. Улучшение вакуума во всем тракте практически на порядок и тщательная работа по выбору пределов изменений уровней СВЧ-мощности на входах не только инжекторной, но и всех секций позволили исключить появление темновых токов на выходе тракта - в месте расположения тестируемых образцов. В дальнейшем, при проведении многочисленных сеансов по калибровке кристаллов пучками предельно малой интенсивности в диапазоне энергий 15-75 МэВ, темновые токи не обнаруживались. Напомним, что темновые токи - это токи на выходе при полностью отключенной пушке. При разогретом катоде электронной пушки электроны на выходе тракта транспортировки в месте расположения исследуемых кристаллов также не были обнаружены регистрирующей аппаратурой.

При получении и регулировке пучка мы применяли комбинацию следующих способов уменьшения интенсивности: понижение температуры термокатода - при этом пушка оставалась в режиме объёмного заряда; коллимация пучка; изменение длительности СВЧ-импульсов в небольших пределах; регулировка интенсивности пучка изменением высокого напряжения на источнике электронов в небольших, заранее найденных пределах, при которых практически не обнаруживается изменение средней энергии потока из-за изменения начальной энергии влета в инжекторную секцию; регулировка элементов, расположенных на неактивных участках ускорителя, где отсутствует ускоряющее поле. Нами использовались разные способы, в зависимости от востребованной выходной интенсивности и энергии; для каждой требуемой выходной энергии электронов выставлялись заранее определенные параметры режима ускорителя, которые затем тщательной подстройкой доводились до оптимальных значений для заданной средней интенсивности одиночных электронов на выходе тракта.

Предварительно пучок настраивался за анализирующим магнитом МА (рис.1) на заданную энергию с интенсивностью несколько десятков нА на цилиндре Фарадея. Пучок через коллиматор с отверстием 3 мм, расположенный на выходе собственно ЛУЭ-75, направлялся в тракт транспортировки. Квадрупольными линзами Q1- Q4 и корректорами (на рис.1 не показаны) за отключенным магнитом M1 на люминофорном экране LS (люминофорное вещество тонким слоем нанесено на каптоновое выходное окно) получалось четкое изображение пучка, а ток прямого пучка измерялся цилиндром Фарадея. Далее включались магниты M1 и M2, и пучок проводился через магнитную оптику тракта параллельного переноса, предварительно отъюстированного лазерным лучом. 
На рис. 2 показана схема эксперимента в зоне расположения исследуемых кристаллов. На выходе тракта формируется пучок со средним током 2-3 нА и диаметром пучкового пятна 3-4 мм. Это то минимальное значение тока, при котором достигается визуальная регистрация профиля пучка на подвижном люминофорном экране, устанавливаемом дистанционно перед выходным окном пучкопровода (рис.2). Подвижный цилиндр Фарадея, кроме измерения тока, обеспечивает во время настройки на люминофоре защиту кристаллов от попадания пучка повышенной интенсивности во избежание их загруженности, исключения влияния послесвечения. После формирования пучкового пятна элементами магнитооптической системы интенсивность пучка понижалась почти до нуля, люминофорный экран и цилиндр Фарадея, преграждавшие путь пучка, дистанционно отодвигались, и интенсивность потока, контролируемая измерительной электроникой, устанавливалась до заданного среднего значения 10-20 электронов в секунду. Одним из критериев выбора интенсивности потока частиц является сведение к минимуму явления наложения сигналов (pile up). С увеличением числа частиц в единицу времени увеличивалось число многоэлектронных событий в секунду. Следовательно, необходимая выходная интенсивность пучка требовала ослабление не менее, чем на 9 порядков.

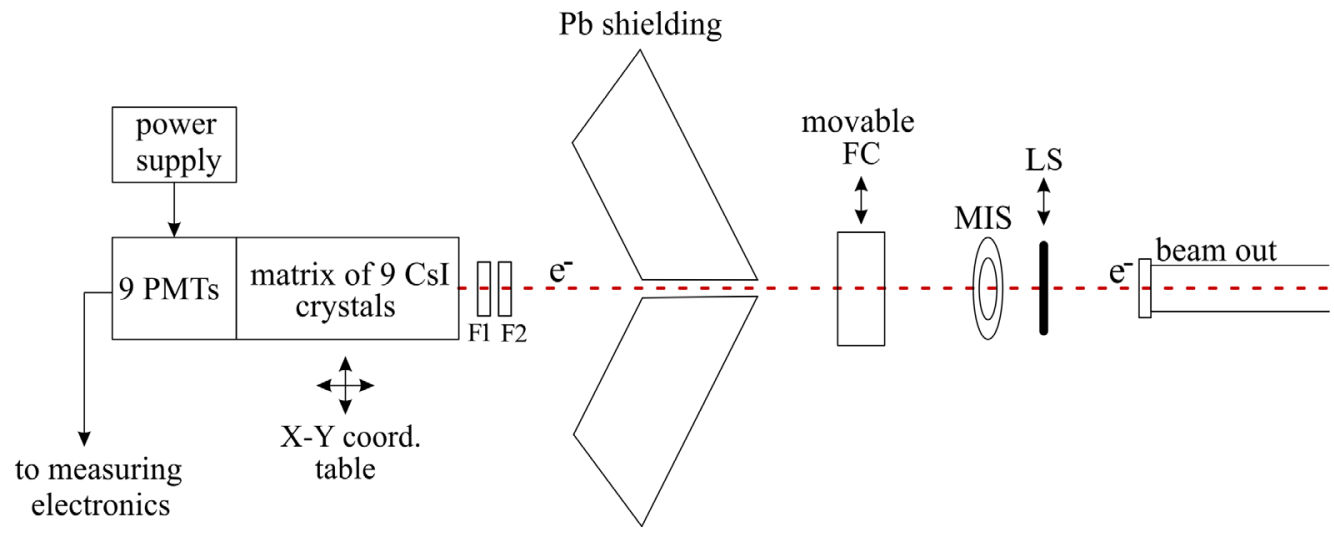

Рис.2. Зона калибровки детекторов. Схема эксперимента.

Энергия и ток ускоренного пучка электронов в той или иной степени зависят от многих настраиваемых параметров ускорителя. Энергия пучка в основном зависит от уровня мощности ускоряющего поля. Имеется также существенная зависимость от величины импульсного тока пучка, выражаемая нагрузочной характеристикой, из которой следовало, что если вводимая в секцию СВЧ-мощность соответствует некоторой максимальной выходной энергии $E_{\max }$ (при $I=0)$, то при предварительной настройке установки на малый ток и дальнейшем уменьшении интенсивности до экстремально низких значений изменение первоначальной энергии частиц будет незначительным. Так, при настройке на средний ток в несколько десятков наноампер и дальнейшем уменьшении интенсивности, изменение средней энергии электронов не превышает $0.1 \mathrm{MэB,} \mathrm{что}$ 
находится в допустимых пределах погрешности измерений.

Дополнительная мощная свинцовая защита толщиной 20 радиационных длин экранирует зону кристаллов от тормозного излучения, возникающего при повороте пучка магнитами параллельного переноса. В защитную стену перед тестируемой матрицей кристаллов встроен довольно протяженный коллиматор, ограничивающий влияние естественной расходимости пучка.

Исследуемая матрица из девяти нелегированных кристаллов CsI (сборка $3 \times 3$ ) снабжена фотоэлектронными умножителями типа ФЭУ-85 (рис.2). Прием, обработка и визуализация данных осуществлялись авторской программой «Маino» одного из соавторов (Г. 3.), позволяющей также запись и хранение результатов для дальнейшей обработки (рис. 3). Программа «Маino» была разработана с использованием классов из ROOT Data Analysis Framework (https://root.cern) и работала в OC Linux. Дистанционно обеспечивался компьютерный мониторинг и управление экспериментом.

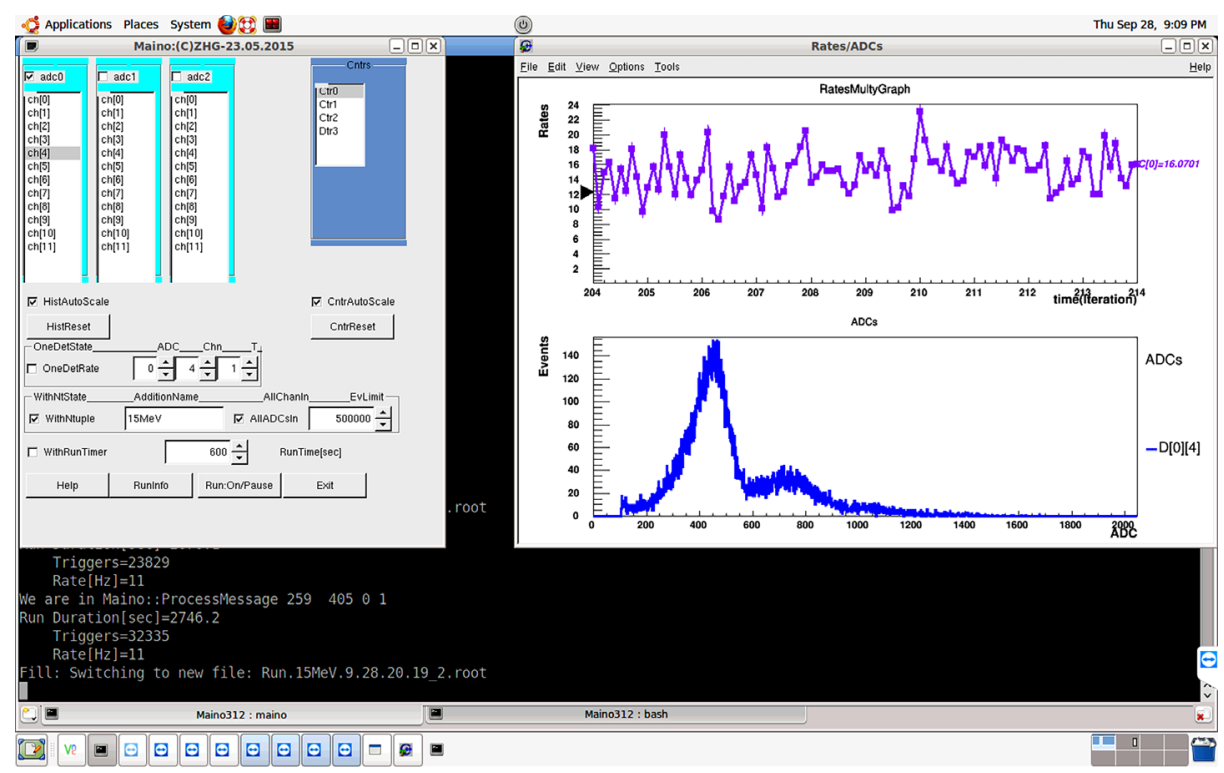

Рис.3. Скриншот интерфейса программы «Маino». Справа: верхний луч строка считывания одиночных событий, внизу - гистограмма распределения числа событий по каналам АЦП при средней энергии электронов 15 МэВ.

\section{3. О других работах, связанных с решением задачи}

Решение описанной научно-методической задачи, впрочем, как и любой другой, требовало создание учитывающей особенности эксперимента соответствующей инфраструктуры, включающей элементы структурной схемы эксперимента, измерительной аппаратуры, программного обеспечения и др. Реализация такой инфраструктуры позволяет утверждать о наличии стенда по решению поставленной и ей подобных задач. 
В связи с востребованным для описанных выше работ энергетическим диапазоном была восстановлена и задействована третья ускорительная станция, благодаря чему энергия электронов была доведена до 75 МэВ [1].

На рис.4 показана осциллограмма высоковольтного импульса модулятора клистрона и огибающая отработанного СВЧ импульса на выходе ускоряющей секции восстановленной третьей ускорительной станции.

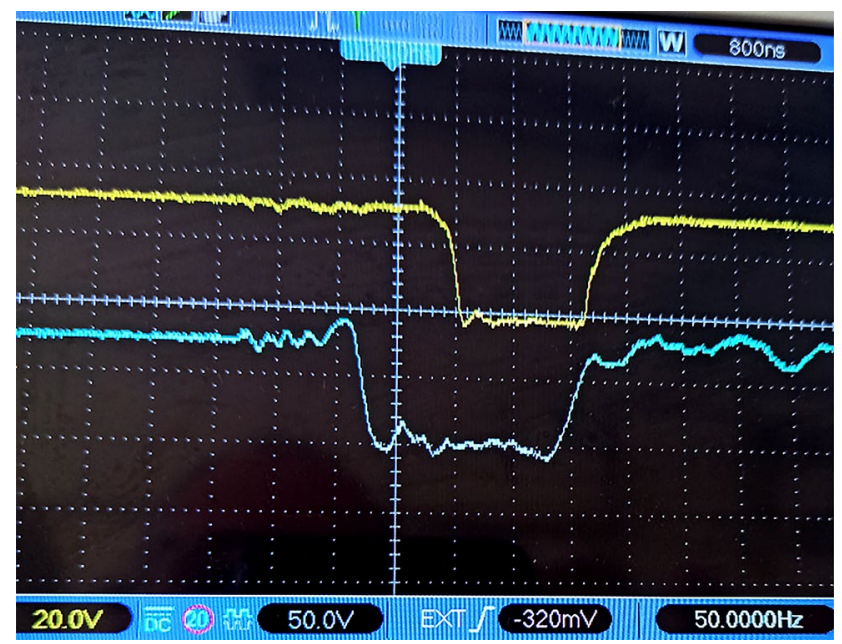

Рис.4. Нижний луч - импульс модулятора; верхний луч - огибающая СВЧ импульса.

Потребовалась также модернизация элементов магнитооптической системы тракта параллельного переноса для работы с пучком в диапазоне энергий 50-75 МэВ. На рис.5 показан один из идентичных поворотных магнитов тракта параллельного переноса после умощнения.

Одновременно с работами по увеличению энергии до 75 МэВ решались задачи и по диагностике пучка. С помощью прокалиброванного магнитного анализатора (МА на рис.1) с цилиндром Фарадея и интегратором тока измеряется энергия и интенсивность пучка за поворотом. Измерение энергии в диапазоне

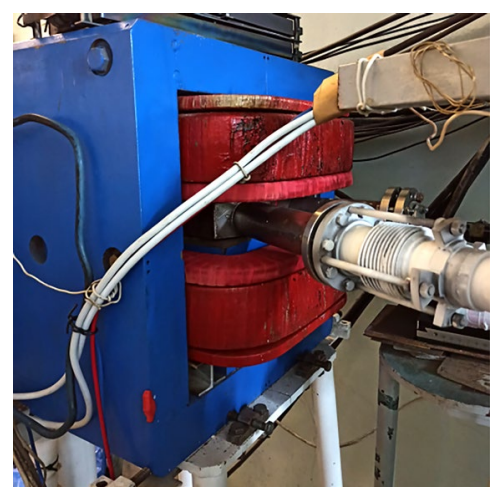

Рис.5. Магнит параллельного переноса с дополнительной обмоткой. 
50-75 МэВ требовало повышенного значения тока через анализирующий электромагнит, поэтому были внесены необходимые изменения в схему питания, регулировки и стабилизации тока электромагнита, а также в систему его охлаждения для исключения возможного перегрева обмоток при повышении их токовой нагрузки.

В процессе эксперимента обеспечивалась возможность неразрушающего непрерывного контроля интенсивности и формы импульса выводного пучка. Мишень устанавливалась перед «прямым пучком» или за поворотным магнитом МА между выходным окном ЛУЭ-75 и цилиндром Фарадея (зоны ЕА на рис.1).

Были рассчитаны и сконструированы магнитоиндукционные датчики, состоящие из тороидального ферритового кольца с 2-мя симметрично намотанными на них обмотками. Наводимые на обмотки сигналы от пучка поступают по двум каналам, включающим двухкаскадные усилители, выполненные на малошумящих быстродействующих ОУ, причем первый каскад каждого канала является дифференциальным усилителем, на выходе которого синфазные помехи вычитаются. Датчики хорошо экранированы для защиты от помех. Таким образом обеспечивается помехозащищенность и высокая чувствительность датчика. Для тестирования датчиков была собрана установка с имитацией тока пучка от генератора импульсов (рис.6).

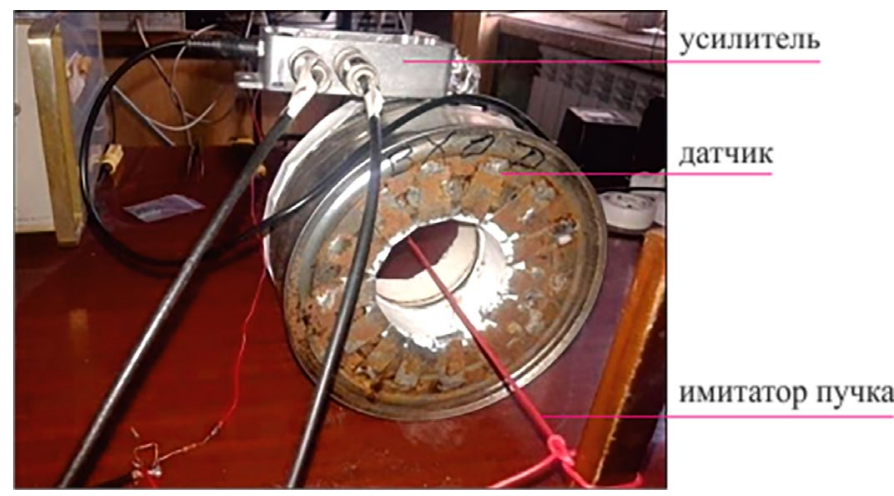

Рис.6. Тестирование датчика с имитацией тока пучка.

Датчики (MIS на рис.2) были установлены на участках выхода пучка в атмосферу в экспериментальных зонах (рис.7). Работа с этими датчиками показала, что их чувствительность гораздо выше, чем у люминофорного экрана, что облегчало обнаружение и настройку низкоинтенсивного пучка при проводке его по тракту.

Для обеспечения стабильности параметров пучка, ускоряющие секции термостатируются с температурной точностью $\pm 0.5^{\circ} \mathrm{C}$. Для компьютерного мониторинга температур различных жизненно важных для ускорителя систем была разработана и внедрена электронная система измерения и контроля с учетом сильного фона электромагнитных помех и наводок в помещениях ускорителя (рис.8). Ток через платиновый терморезистор типа Pt100, линейно зависящий от 


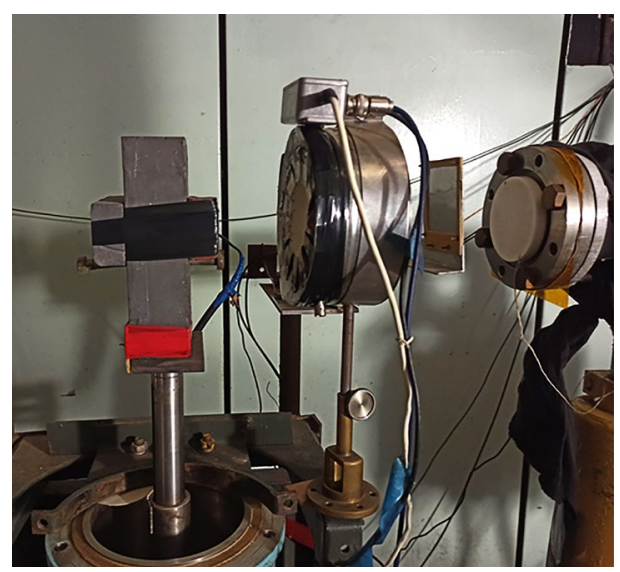

Рис.7. Магнитно-индукционный датчик с усилителем на выходе пучкопровода.

температуры термостатирующей воды, пропорционально преобразуется в частоту следования импульсов, передаваемых по коаксиальному кабелю на пульт управления ЛУЭ-75 и поступающих на пересчетное устройство в стандарте CAMAC.

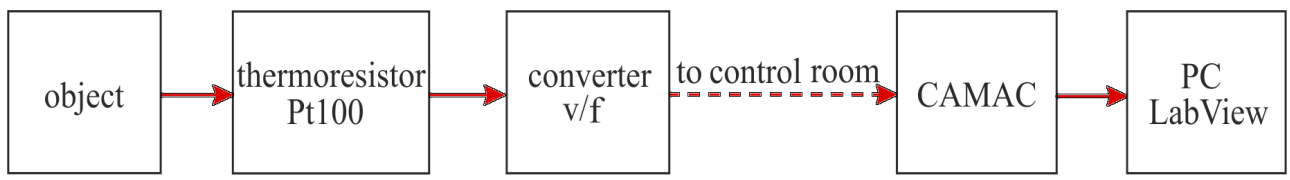

Рис.8. Блок-схема измерения температуры.

Показания температуры термостатирующей воды ускоряющих секций и охлаждающей воды на входах-выходах мощных клистронов выведены на монитор компьютера, где обработка и визуализация информации осуществляются с помощью программы LabView.

Был сконструирован координатный стол (рис.2) для размещения на нем тестовой матрицы кристаллов при облучении пучком электронов, и подготовлено программное обеспечение для управления его перемещением. Координатный стол позволяет с высокой точностью сканировать сборку кристаллов в поперечной плоскости посредством компьютера как непосредственно в зале ускорителя, так и из комнаты экспериментаторов, где осуществляется управление ходом эксперимента с помощью одной из программ удаленного доступа к компьютеру.

\section{4. Заключение}

Наличие тракта параллельного переноса пучка, низкий уровень темновых токов и правильный выбор режимов работы систем ускорителя с учетом токовой нагрузки позволили создать благоприятные условия для получения электронных пучков экстремально низкой интенсивности. 
Совместные успешные работы исследовательских групп Отдела экспериментальной физики ННЛА (Ереван) и Лаборатории ядерных проблем ОИЯИ (Дубна) показали, что линейный ускорительный комплекс ЛУЭ-75 может служить источником тестовых пучков экстремально низкой интенсивности для калибровки детекторов элементарных частиц. Как отмечалось во введении, такие низкоинтенсивные пучки могут быть использованы для решения различных задач в области ядерной физики низких энергий. Известны работы по облучению наночастиц радионуклидами [9], но представляется интересным также испытание нанотрубок на радиационную стойкость при более высоких энергиях пучками низкой интенсивности.

В настоящее время ускоритель работает надежно. Считаем целесообразным проведение работ по модернизации отдельных узлов и систем с внедрением, где это возможно, цифровой техники с целью дальнейшего повышения эффективности. В первую очередь, это относится к системам обеспечения ускорителя: системе термостатирования и охлаждения, электро-радиотехнических компонентов, вакуумной системе, системе управления.

Авторы выражают благодарность профессорам Лазиеву Э.М. и Будагову Ю.А. за инициирование данной работы по получению описанных режимов линейного ускорителя, их практическому применению и постоянное внимание к ней.

\section{ЛИТЕРАТУРА}

1. A.M. Sirunyan, A.S. Hakobyan, A.Z. Babayan, H.H. Marukyan, H.G. Mkrtchyan, K.D. Davtyan, H.L. Arutyunov, G.M. Ayvazyan, S.K. Avagyan, V.H. Martirosyan, A.A. Margaryan, G.G. Khachatryan, L.R. Vahradyan. J. Contemp. Phys., 54, 225, (2019).

2. A.Y. Aleksanyan, S.M. Amirkhanyan, A. Balabekyan, N.A. Demekhina, H.R. Gulkanyan, T.V. Kotanjyan, V. Mangasaryan, V.S. Pogosov, L.A. Poghosyan, S. Faltajanyan. J. Contemp. Phys., 55, 431 (2020).

3. L. Bartoszek et al. arXiv:1501.05241, 2015.

4. М.Н. Хачатурян. Физика элементарных частиц и атомного ядра (ЭЧАЯ), 34(5), 1316 (2003).

5. С.А. Бунятов, А.В. Красноперов, Ю.А. Нефедов, Б.А. Попов, В.В. Терещенко, Н.И. Божко, А.С. Вовенко, В.Н. Горячев, Ю.И. Соломатин, В.А. Сенько, А.В. Сидоров. Письма в ЭЧАЯ, 4(6) 847 (2007).

6. R. Taniguchi, T. Kojima, S. Okuda. Radiation Physics and Chemistry, 76, 1779 (2007).

7. L.D. Yu, J.H. Yue, Y.L. Li, Y.F. Sui. Proceedings of IPAC2017, Copenhagen, Denmark, 292 (2017).

8. Л. Биберман, Н.Сушкин, В.Фабрикант. УФН, 38, 570 (1949).

9. N.P. Dikiy, A.N. Dovbnya, Yu.V. Lyashko, et al., PROBLEMS OF ATOMIC SCIENCE AND TECHNOLOGY, no. 5(105), 83 (2016). 


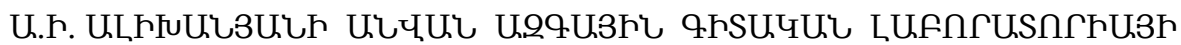

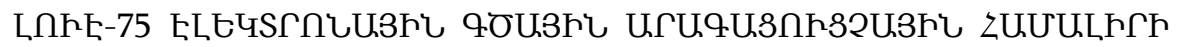

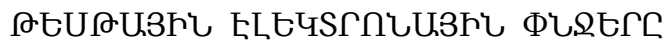

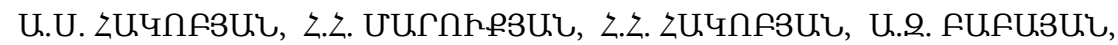

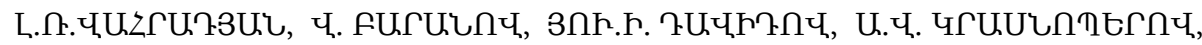

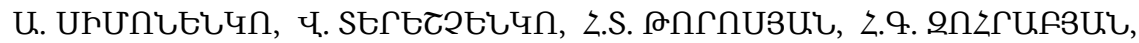

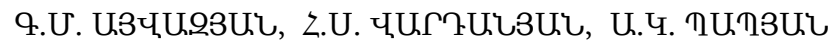

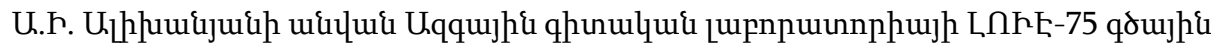

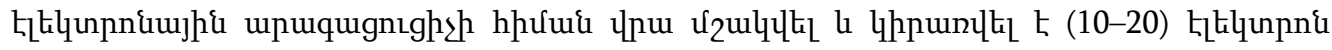

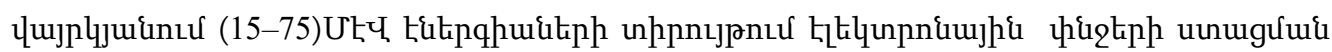

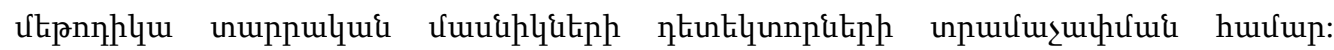

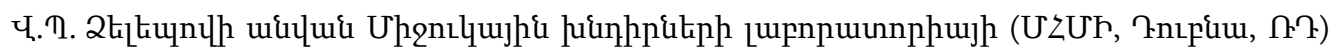

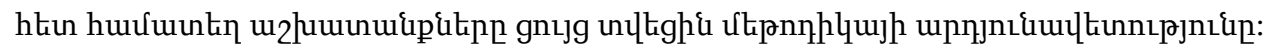

\section{TEST ELECTRON BEAMS BASED ON THE LINEAR ACCELERATOR COMPLEX LUE-75 OF A.I. ALIKHANYAN NATIONAL SCIENTIFIC LABORATORY}

A.S. HAKOBYAN, H.H. MARUKYAN, H.H. HAKOBYAN, A.Z. BABAYAN, L.R. VAHRADYAN, V. BARANOV, Yu.I. DAVYDOV, A. KRASNOPEROV, A. SIMONENKO, V. TERESHCHENKO, H.T. TOROSYAN, H.G. ZOHRABYAN, G.M. AYVAZYAN, H.S. VARDANYAN, A.K. PAPYAN

Based on the linear electron accelerator LUE-75 of the A.I. Alikhanyan National Scientific Laboratory a technique for obtaining controlled primary electron beams with an intensity of (10-20) electrons per second in the energy range (15-75) MeV for elementary particle detectors calibration was developed and applied. Joint work with the V.P. Dzhelepov Laboratory of Nuclear Problems (JINR, Dubna, RF) showed the efficiency of the technique. 